\title{
Motion Induced Coherence Degradation in Passive Systems
}

\author{
ALBERT A. GERLACH, SENIOR MEMBER, IEEE
}

\begin{abstract}
The problem of correlation degradation introduced as a result of the track and motional instabilities in a transiting target is addressed. The target is assumed to consist of a narrow-band signal source which is received at two remotely located sensors. The outputs are processed through a correlation-type detector.

The correlator output will, in general, suffer a degradation which is nondeterministic and statistical in character. Two important aspects of the target motion which can significantly degrade the signal coherence are, the mean target track relative to the source-sensor geometry, and the motional instabilities relative to the mean target track. The former creates an absolute upper bound on the useful coherence integration time which can be employed in the processor. The latter will depend on the spectral characteristics of the motional instabilities as well as the variance of the target course and speed. Results of the expected processor degradation are presented in both functional and graphical form for convenience in interpretation and data abstraction. An optimum integration time is derived from the standpoint of optimizing the processor gain for signal detection in an incoherent signal background.

It is concluded that target motion can be the most serious deterrent to the use of coherent signal processors over exceptionally long integration intervals.
\end{abstract}

\section{INTRODUCTION}

$\mathrm{C}$ OHERENT signal processing is a proven technique for detection and parameter estimation in a number of practical applications. To date, however, little is known about the limitations on the integration interval over which coherent processing can be usefully employed.

The "subject paper addresses the problem of correlation degradation introduced as a result of the track and motional anomalies inherent in a transiting target. The target is assumed to emit a narrowband signal signature which is being received at two or more remotely located signal sensors. The normal signal compensation controls available in correlation processing are; a fixed time delay (to align the two received signals in time register), and a fixed time scale-factor shift (to compensate for Doppler differences between the two signals). Although these controls have proven adequate over sufficiently small integration intervals, the correlation degradation inherent in target motion will become increasingly severe as the analysis interval (or integration time) is extended.

It will be the object of this paper to determine, quantitatively, the correlation degradation (due to target motion considerations) to be expected as a function of; the system geometry, the signal center frequency, the correlation integration time, and the nature and characteristics of the target motional track.

Manuscript received December 15, 1976. This work was supported by the Defense Advanced Research Projects Agency under Grant ARPA 2275.

The author is with the Naval Research Laboratory, Washington, DC 20375.
For a given system geometry, signal center frequency, and anticipated motional characteristics then, an appropriate integration time can be chosen to optimize the correlation processor in some useful sense. To this end, the analyses in this paper are objectively conducted.

\section{Formulation of the Problem}

\section{A. Time Scale-Factor Function}

When a signal source is in motion in an ideal transmission medium, the signal received at a remote point in the medium will be a distorted replica of the source signal. If the source signal is $u(t)$, and if we ignore attenuation and the propagation delay time, the signal received at a remote sensor $x$ can be written as $u\left(k_{x} t\right)$, where $k_{x}$ is the time scale-factor distortion due to source motion. ${ }^{1}$ The time scale-factor function $k_{x}$ will obey the simple dynamics relation

$$
\begin{aligned}
d\left(k_{x} t\right) & =d t+\frac{v_{x}}{c} d t \\
& =\left(1+\frac{v_{x}}{c}\right) d t
\end{aligned}
$$

where $v_{x}$ is the component of source velocity along the propagation path from the source to the receiving sensor, and $c$ is the average velocity of propagation over the propagation path. For convenience, the time scale-factor function may be written as

$$
k_{x}(t)=1+\delta_{x}(t)
$$

where $\delta_{x}$ is called the time scale-factor shift between the source and receiving sensor. Thus, it is seen that $\delta_{x}$ is the solution to the simple first order differential equation

$$
\frac{d}{d t}\left(\delta_{x} t\right)=t \frac{d \delta_{x}}{d t}+\delta_{x}=\frac{v_{x}}{c} .
$$

It may be verified that the solution to the above equation is

$$
\delta_{x}=\frac{1}{t} \int_{a}^{t} \frac{v_{x}}{c} d \tau \equiv\left\langle v_{x} / c\right\rangle_{t, a}
$$

where $a$ is an arbitrary constant. From (2-4), it is evident that the time scale-factor shift function is a form of running time average of $v_{x} / c$ involving an arbitrary constant. Consequently, when $v_{x} / c$ is invariant (constant), $\delta_{x}$ may be reduced to a constant equal to $v_{x} / c$.

Worthy of note at this point is that the ratio of $v_{x}$ to $c$ is

\footnotetext{
${ }^{1}$ See the Appendix.
} 
typically a very small number for most problems of practical interest. Consequently, in the analyses to follow, it shall always be assumed that $\left|v_{x}\right|<<c$. Furthermore, in the analyses to follow, the propagation velocity $c$ will be treated as a constant. This, in reality, represents no serious restriction on the problem, since $c$ could readily be written as $c=c_{o}(1+\Delta)$; where $c_{o}$ is the mean value of $c$ and $\Delta c_{0}$ represents the variable component of $c$. The factor, $1 /(1+\Delta)$, can then be considered as belonging to the $v_{x}$ factor. That is, the factor $v_{x}$ (in reality) represents the function $v_{x} /(1+\Delta)$.

\section{B. Geometry Considerations}

Consider the system geometry illustrated in Fig. 1. A target source, located at point 0 , is traveling along some course $\beta$ at a velocity $v$ (either or both of which may be time variable). The ray paths from the source to the receiving points $x$ and $y$ emanate along courses $\beta_{o x}$ and $\beta_{o y}$, respectively.

From $(2-4)$, the time scale-factor shift variables realized at the $x$ and $y$ receiving sensors will be

$$
\begin{aligned}
& \delta_{x}=\left\langle\frac{v}{c} \cos \left(\beta_{o x}-\beta\right)\right\rangle_{t, a} \\
& \delta_{y}=\left\langle\frac{v}{c} \cos \left(\beta_{o y}-\beta\right)\right\rangle_{t, a}
\end{aligned}
$$

where all of the individual elements in the above expressions can be considered as variable.

The difference in time scale-factor shift that will exist between the two receiving sensors $y$ and $x$, will therefore be

$$
\begin{aligned}
& \delta_{y x}\left(v, \beta ; \beta_{o x}, \beta_{o y}\right)=\delta_{y}-\delta_{x} \\
& =\left\langle\frac{v}{c}\left[\cos \left(\beta_{o y}-\beta\right)-\cos \left(\beta_{o x}-\beta\right)\right]\right\rangle_{t, a} \\
& =\left\langle 2 \frac{v}{c} \sin \frac{1}{2} \alpha \sin \varphi\right\rangle_{t, a}
\end{aligned}
$$

where

$$
\begin{aligned}
& \alpha=\beta_{o x}-\beta_{o y} \\
& \varphi=\frac{1}{2}\left(\beta_{o x}+\beta_{o y}\right)-\beta .
\end{aligned}
$$

The parameter $\alpha$ is simply the aperture angle from the source to the two receiving sensors, and the parameter $\varphi$ is a measure of the source course relative to the mean angle from the source to the two receiving sensors.

\section{Target Motion Considerations}

Assume, now, that the relative ranges illustrated in Fig. 1 are sufficiently large so that systematic changes in the relative geometry can be ignored over the temporal intervals which will be considered in the analysis to follow. (This presents no serious restriction since the systematic geometry variation problem can be treated separately in a manner similar to the problem being presently formulated.) Under the stipulated restrictions, the parameter $\beta_{o x}$ and $\beta_{o y}$ will be considered constant.

It shall be assumed that the source velocity $v$ and course $\varphi$ are independent variables which vary in some manner about their individual mean values. Let

$$
v=v_{0}+\sigma_{v} \xi_{1}
$$

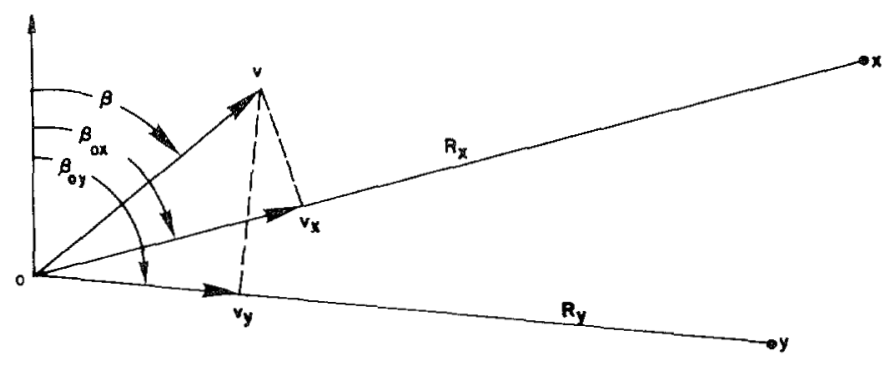

Fig. 1. Vector geometry of the problem.

and

$$
\varphi=\varphi_{0}+\sigma_{\varphi} \xi_{2} \text { (radians) }
$$

where $v_{0}$ and $\varphi_{0}$ are the mean values of $v$ and $\varphi$, respectively, $\sigma_{v}$ and $\sigma_{\varphi}$ are the standard deviations of the respective variables about their mean values, and $\xi_{1}$ and $\xi_{2}$ represent normalized, zeromean, dynamic variables whose standard deviations are equal to unity. For purposes of the subject analysis, it shall be assumed that $\sigma_{v} \xi_{1}<<v_{0}$ and $\sigma_{\varphi} \xi_{2}<<1$ radian.

Since now,

$$
\begin{aligned}
\sin _{\varphi} & =\sin \left(\varphi_{0}+\sigma_{\varphi} \xi_{2}\right) \\
& \approx \sin \varphi_{0}+\sigma_{\varphi} \xi_{2} \cos \varphi_{0}
\end{aligned}
$$

(2-6) may be approximated as

$$
\begin{aligned}
\delta_{y x}= & \left\langle2 \frac { v _ { 0 } } { c } \operatorname { s i n } \frac { 1 } { 2 } \alpha \left[\sin \varphi_{0}+\left(\frac{\sigma_{v}}{v_{0}} \sin \varphi_{0}\right) \xi_{1}\right.\right. \\
& \left.\left.+\left(\sigma_{\varphi} \cos \varphi_{0}\right) \xi_{2}\right]\right\rangle_{t, a} \\
= & 2 \frac{v_{0}}{c} \sin \frac{1}{2} \alpha\left[\sin \varphi_{0}+\frac{\sigma_{v}}{v_{0}} \sin \varphi_{0}\left\langle\xi_{1}\right\rangle_{t, a}\right. \\
& \left.+\sigma_{\varphi} \cos \varphi_{0}\left\langle\xi_{2}\right\rangle_{t, a}\right] .
\end{aligned}
$$

For convenience, new variables $\zeta_{1}$ and $\zeta_{2}$ will be defined as follows. Let

$$
\begin{aligned}
\zeta_{i}(t) & =t\left\langle\xi_{i}\right\rangle_{t, a}, \quad(i=1,2) \\
& =\int_{0}^{t} \xi_{i}(\tau) d \tau-K_{i}
\end{aligned}
$$

where $K_{i}$ is a state variable chosen to make $\zeta_{i}(t)$ a zero mean variable over the analysis interval $T$. Thus,

$$
\begin{aligned}
K_{i} & =\int_{0}^{a} \xi_{i}(\tau) d \tau \\
& =\frac{1}{T} \int_{0}^{T}\left[\int_{0}^{t} \xi_{i}(\tau) d \tau\right] d t .
\end{aligned}
$$

From (2-11) and (2-12) then

$$
\delta_{y x}-\hat{\delta}_{y x}=2 \frac{v_{0}}{c} \sin \frac{1}{2} \alpha\left[\left(\frac{\sigma_{v}}{v_{0}} \sin \varphi_{0}\right) \frac{\zeta_{1}}{t}+\left(\sigma_{\varphi} \cos \varphi_{0}\right) \frac{\zeta_{2}}{t}\right]
$$


where

$$
\hat{\delta}_{y x}=\frac{2}{T}\left\langle t \delta_{y x}\right\rangle_{T}=2 \frac{v_{0}}{c} \sin \frac{1}{2} \alpha \sin \varphi_{0}
$$

is defined to minimize the correlation degradation over the analysis interval $T$ by ensuring that the mean value of $\left(\delta_{y x}-\right.$ $\left.\hat{\delta}_{y x}\right) t$ over the integration time $T$ is zero.

From (2-14), it can be seen that the coefficients of the two terms are in quadrature relative to the mean course parameter $\varphi_{0}$. Thus, when the one coefficient is maximum, the other will be zero. As will become evident shortly, (2-14) will play a significant role in the analyses to follow.

\section{Time Scale-Factor Correction}

Assume now that the target signals, received at the $x$ and $y$ receiving sensors (see Fig. 1), have been appropriately aligned in time register at some fixed reference point in time (say at $t=0$ ). The two received signals will then differ only in time scale-factor. The two signals can be written as $u_{x}(t)=u\left(k_{x} t\right)$ and $u_{y}(t)=u\left(k_{y} t\right)$, respectively; where $k_{x}=1+\delta_{x}$ and $k_{y}=1+\delta_{y}$. Therefore,

$$
u_{y}(t)=u_{x}\left\{\left(k_{y} / k_{x}\right) t\right\} \approx u_{x}\left\{\left(1+\delta_{y x}\right) t\right\}
$$

since

$$
\frac{k_{y}}{k_{x}}=\frac{1+\delta_{y}}{1+\delta_{x}}=1+\delta_{y x}\left[1+\sum_{n=1}^{\infty}\left(-\delta_{x}\right)^{n}\right] .
$$

Because $\delta_{y x}$ is variable, it cannot be compensated for perfectly by a simple fixed time scale-factor change. However, $\delta_{y x}$ can be compensated to reduce the correlation degradation between the two signals to a minimum. Correcting for the optimum time scale-factor shift then,

$$
u_{y}\left\{\left(1-\hat{\delta}_{y x}\right) t\right\} \approx u_{x}\left\{\left(1+\delta_{y x}-\hat{\delta}_{y x}\right) t\right\}
$$

where $\delta_{y x}-\hat{\delta}_{y x}$ is given in $(2-14)$.

\section{E. The Correlation Integral}

The relevant cross-correlation function will involve the product of the signals from the two receiving sensors after the signals have been adjusted in both time register and time scalefactor. Consequently, when the two signals are properly aligned in time register and corrected for the optimum time scale-factor shift, the product function will be

$$
u_{x}(t) u_{y}\left\{\left(1-\hat{\delta}_{y x}\right) t\right\} \approx u_{x}(t) u_{x}\left\{\left(1-\delta_{y x}-\hat{\delta}_{y x}\right) t\right\} \text {. }
$$

Assuming that $u_{x}(t)$ is a narrow bandwidth signal, it can be represented as

$$
u_{x}(t)=\sin (2 \pi f t+\phi)
$$

where $f$ is a parametric variable ${ }^{2}$ with mean value $f_{c}$, and whose maximum deviation about $f_{c}$ is small compared with $f_{c}$. Under these circumstances, the product function may be written as

\footnotetext{
${ }^{2} \mathrm{~A}$ parametric variable, in this case, is one which may be treated as a parameter from the short-term standpoint (time in the order of one to several times $1 / f_{c}$ ), but can otherwise vary over long time intervals. The parametric restriction is, primarily, on the rate-of-change of the variable.
}

$$
\begin{aligned}
u_{x}(t) u_{y}\left\{\left(1-\hat{\delta}_{y x}\right) t\right\} \approx & \sin (2 \pi f t+\phi) \\
& \cdot \sin \left[2 \pi f\left(1+\delta_{y x}-\hat{\delta}_{y x}\right) t+\phi\right] \\
= & \frac{1}{2}\left\{\cos \left[2 \pi f\left(\delta_{y x}-\hat{\delta}_{y x}\right) t\right]\right. \\
& \left.-\cos \left[2 \pi f\left(2+\delta_{y x}-\hat{\delta}_{y x}\right) t+2 \phi\right]\right\}
\end{aligned}
$$

And the resulting normalized cross-correlation function, over integration time $T$ (assuming that $T$ is very large compared with $1 / f_{c}$ ), becomes

$$
\gamma_{N}(T) \approx \frac{1}{T} \int_{0}^{T} \cos \left[2 \pi f_{c}\left(\delta_{y x}-\hat{\delta}_{y x}\right) t\right] d t
$$

When $\delta_{y x}-\hat{\delta}_{y x}$ is zero (that is, when $\sigma_{v}$ and $\sigma_{\varphi}$ are both zero), the resulting correlation function is simply unity. Consequently, $\gamma_{N}(T)$ will be a measure of the degradation in correlation coefficient suffered as a result of the instabilities in target (source) motion.

\section{SOLUTION OF THE CORRELATION DEgRADATION FUNCTION}

\section{A. Definition of Parameters and Variables}

Before solving the correlation degradation function, it will be convenient to define new parameters as follows. Let

$$
\epsilon_{1}=2 f_{c}\left(o_{v} / c\right) \sin \frac{1}{2} \alpha \sin \varphi_{0}
$$

and

$$
\epsilon_{2}=2 f_{c}\left(v_{0} \sigma_{\varphi} / c\right) \sin \frac{1}{2} \alpha \cos \varphi_{0}
$$

From (2-14), (3-1), and (3-2) then, the phase argument in (2-22) may be written

$$
\begin{aligned}
\theta(t) & =2 \pi f_{c}\left(\delta_{y x}-\hat{\delta}_{y x}\right) t \\
& =2 \pi\left[\epsilon_{1} \zeta_{1}(t)+\epsilon_{2} \zeta_{2}(t)\right] .
\end{aligned}
$$

To solve a specific problem, each term in (3-3) can be treated separately and then summed to form the composite phase function, $\theta(t)$. It will be convenient, however, to define a single variable and a single parameter as follows. Let

$$
\epsilon \zeta(t)=\epsilon_{1} \zeta_{1}(t)+\epsilon_{2} \zeta_{2}(t)
$$

where

$$
\epsilon=\sqrt{\epsilon_{1}^{2}+\epsilon_{2}^{2}} \text {. }
$$

The variance of the new variable will be

$$
\overline{\zeta^{2}}=\frac{1}{\epsilon^{2}}\left[\epsilon_{1}^{2} \overline{\zeta_{1}^{2}}+\epsilon_{2}^{2} \overline{\zeta_{2}^{2}}+2 \rho \epsilon_{1} \epsilon_{2} \sqrt{\overline{\zeta_{1}^{2}} \overline{\zeta_{2}^{2}}}\right]
$$

where $\rho$ is the correlation coefficient between the two variables, $\zeta_{1}(t)$ and $\zeta_{2}(t)$. When the indicated variables are statistically independent or are orthogonal, the last term in (3-6) will be zero. In the revised nomenclature,

$$
\theta(t)=2 \pi \epsilon \zeta(t)
$$

and

$$
\sigma_{\theta}(T)=2 \pi \epsilon \sqrt{\overline{\xi^{2}}}=2 \pi \epsilon \sigma_{\zeta}(T) .
$$




\section{B. Solution Dependence on $p_{\theta}(\theta)$ and $\sigma_{\theta}(T)$}

From (2-22) and (3-3), the correlation degradation function becomes

$$
\begin{aligned}
\gamma_{N}(T) & =\frac{1}{T} \int_{0}^{T} \cos [\theta(t)] d t=\int_{-\infty}^{\infty} p_{\theta}(\theta) \cos \theta d \theta \\
& =1-\frac{1}{2} \sigma_{\theta}^{2}(T)+\sum_{n=2}^{\infty}(-1)^{n} \frac{m_{2 n}(T)}{(2 n) !}
\end{aligned}
$$

where $p_{\theta}(\theta)$ is the probability density, $\sigma_{\theta}^{2}(T)$ is the variance, and $m_{k}(T)$ is the $k$ th moment of $\theta(t)$ all computed over the interval $0 \leqslant t \leqslant T$.

Consider, now, that $\xi_{1}(t)$ and $\xi_{2}(t)$ are random Gaussian variables. Under these circumstances, $\zeta(t)$ and $\theta(t)$ will be zero mean statistical variables with a Normal probability density distribution. Consequently, $\gamma_{N}(T)$ is readily determined to be

$$
\begin{aligned}
\gamma_{N}(T) & =\sqrt{\frac{2}{\pi}} \int_{0}^{\infty} e^{-(1 / 2) x^{2}} \cos \left(\sigma_{\theta} x\right) d x \\
& =e^{-(1 / 2) \sigma_{\theta}^{2}}
\end{aligned}
$$

Consider next that $\zeta(t)$ is a sinusoidal type of function whose amplitude and frequency may be slowly varying. The probability density for this type of function may be shown to be

$$
p_{\theta}(\theta)=\frac{1}{\pi} \int_{|\theta|}^{\infty} \frac{p_{R}(x) d x}{x \sqrt{1-(\theta / x)^{2}}}
$$

where $p_{R}(x)$ is the probability density of the sinusoidal amplitude variable. Therefore, from (3-9)

$$
\begin{aligned}
\gamma_{N}(T) & =\frac{1}{\pi} \int_{-\infty}^{\infty} \int_{|\theta|}^{\infty} \frac{p_{R}(x) \cos \theta}{x \sqrt{1-(\theta \mid x)^{2}}} d x d \theta \\
& =\frac{2}{\pi} \int_{0}^{\infty} p_{R}(x) \int_{0}^{1} \frac{\cos (x y)}{\sqrt{1-y^{2}}} d y d x \\
& =\int_{0}^{\infty} p_{R}(x) J_{0}(x) d x
\end{aligned}
$$

where $J_{0}(x)$ is the zero-order Bessel function of the first kind for the indicated argument.

When $p_{R}(x)$ is the Rayleigh distribution function, (3-12) reduces to (3-10) as would be expected (since, in this case, $\theta$ will be Normally distributed). When the sinusoidal amplitude is constant (equal to $\theta_{M}$ ), (3-12) becomes simply

$$
\gamma_{N}(T)=J_{0}\left(\theta_{M}\right)
$$

And, if the amplitude is uniformly distributed over the range of $0 \leqslant \theta \leqslant \theta_{M},(3-12)$ reduces to

$$
\gamma_{N}(T)=\frac{1}{\theta_{M}} \int_{0}^{\theta_{M}} J_{0}(x) d x=\frac{2}{\theta_{M}} \sum_{k=0}^{\infty} J_{2 k+1}\left(\theta_{M}\right)
$$

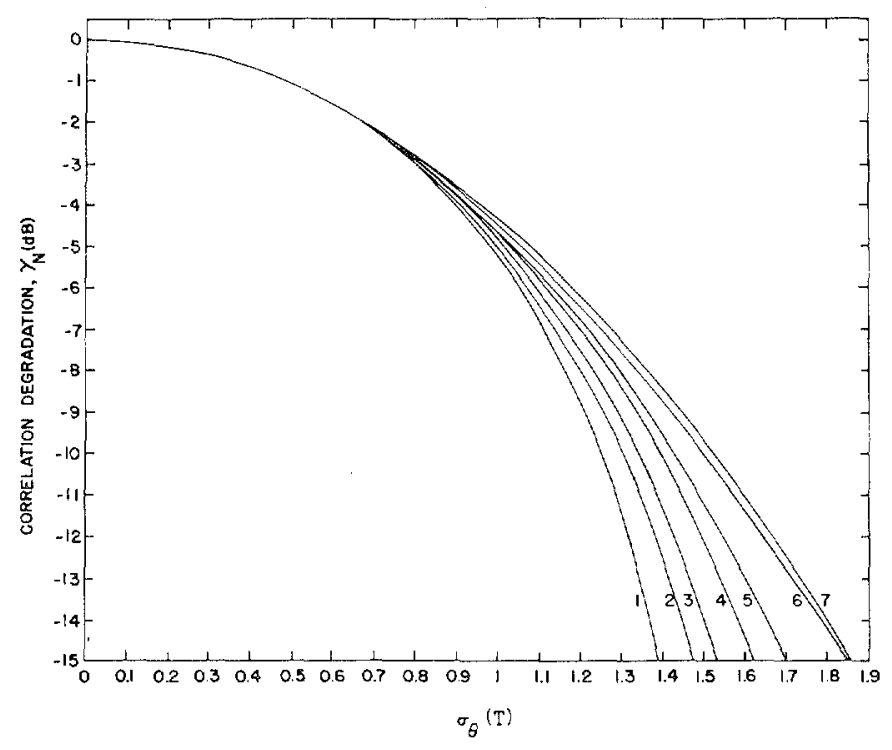

Fig. 2. Correlation degradation as a function of $p_{\theta}(\theta)$ and $\sigma_{\theta}$.

To study the effect of a variety of phase distributions on the correlation degradation, (3-9) has been computed for seven different (but symmetrical) phase probability density functions. The results are illustrated in Fig. 2. The individual curves are a plot of the correlation degradation $\gamma_{N}(T)$, as a function of the standard deviation $\sigma_{\theta}$ for each phase distribution. The probability density functions chosen, along with the output solutions, are given in Table I.

The seven probability density functions chosen span a gamut of phase concentrations from the extreme limits $\pm \theta_{M}$ to near zero. In Curve 1, the phase is concentrated totally at the outer extremity limits, while in Curve 2 the phase distribution is biased toward the outer extremity limits $\pm \theta_{M}$. Curve 3 is the result of a uniform phase distribution over $-\theta_{M} \leqslant \theta \leqslant \theta_{M}$. In curves 4,5 , and 6 , the phase distributions become more concentrated toward the central (or zero) position and decrease to zero at the extremity limits $\pm \theta_{M}$. Curve 7 is the result of a Normal (or Gaussian) probability density function with zero mean.

The significant feature of Fig. 2 is the fact that for values of $\sigma_{\theta}$ less than or equal to about unity, the correlation degradation is (almost) totally dependent on only the value of the standard deviation $\sigma_{\theta}$ and (relatively) independent of the phase distribution. For purposes of analysis, this is important. It means that knowledge of the standard deviation $\sigma_{\theta}$ alone is sufficient to accurately predict the amount of correlation degradation due to motional trajectory anomalies. This will be true just as long as such degradation predictions are less than about 4 to $5 \mathrm{~dB}$ ( $\sigma_{\theta}$ equal to or less than unity). As the correlation degradation increases beyond these lower bounds, one is less inclined to be concerned with the precision of the prediction since, in general, the degradation has reached what can be considered intolerable levels. In any event, the range of correlation degradation is certainly evident from the cluster of curves in Fig. 2.

As a consequence of the above analysis, it is reasonable to bound the correlation coefficient as follows

$$
\cos \left(\sigma_{\theta}\right) \leqslant \gamma_{N}(T) \leqslant e^{-\sigma_{\theta}^{2} / 2} .
$$


TABLE I

Probabilurty Density and Associated Correlation

Degradation Functions

\begin{tabular}{|c|c|c|c|}
\hline & $p(\theta),-\theta_{M} \leqslant \theta \leqslant \theta_{M}$ & $\hat{O}_{M} / 0_{0}$ & $\gamma_{N}(T)$ \\
\hline 1 & $\frac{1}{2}\left[\delta\left(\theta+\theta_{M}\right)+\delta\left(\theta-\theta_{M}\right)\right]$ & 1 & $\cos \theta_{M}$ \\
\hline 2 & $\frac{1}{\pi \sqrt{\theta_{M}^{2}-\theta^{2}}}$ & $\sqrt{2}$ & $J_{0}\left(\theta_{M}\right)$ \\
\hline 3 & $\frac{1}{2 \theta_{M}}$ & $\sqrt{3}$ & $\frac{\sin \theta_{M}}{\theta_{M}}$ \\
\hline 4 & $\frac{\pi}{4 \theta_{M}} \cos \left(\pi \theta / 2 \theta_{M}\right)$ & $\frac{\pi}{\sqrt{\pi^{2}-8}}$ & $\frac{\pi^{2} \cos \theta_{M}}{\pi^{2}-40_{M}^{2}}$ \\
\hline 5 & $\frac{\theta_{M}-10 \mid}{\theta_{M}^{2}}$ & $\sqrt{6}$ & $\frac{4 \sin ^{2}\left(\theta_{M} / 2\right)}{0_{M}^{2}}$ \\
\hline 6 & $\frac{1}{\pi \theta_{M}} \ln \left[\frac{\theta_{M}+\sqrt{\theta_{M}^{2}-02}}{|\theta|}\right.$ & $\sqrt{6}$ & $\frac{1}{M_{0}} \int_{0}^{O_{M}} J_{0}(x) d x$ \\
\hline 7 & $\frac{1}{\sqrt{2 \pi} U_{0}} e^{-\sigma^{2} / 2 \sigma_{\theta}^{2}}$ & - & $e^{-0.02 / 2}$ \\
\hline
\end{tabular}

The problem of determining the standard deviating $\sigma_{\theta}$, from a knowledge of the characteristics of the variable $\xi(t)$ will now be addressed.

\section{Determination of Standard Deviation $\sigma_{\theta}(T)$}

As defined earlier, the motional variable $\xi(t)$ is a zero mean function with unity variance over the analysis interval $0 \leqslant t \leqslant T$. Thus, over the analysis interval $T$, this function can be represented by the Fourier series

$$
\xi(t)=2 \sum_{1}^{\infty}\left[A_{c n} \cos (2 n \pi t / T)+A_{s n} \sin (2 n \pi t / T)\right]
$$

where

$$
A_{c n}=\frac{1}{T} \int_{0}^{T} \xi(t) \cos (2 n \pi t / T) d t
$$

and

$$
A_{s n}=\frac{1}{T} \int_{0}^{T} \xi(t) \sin (2 n \pi t / T) d t .
$$

And the variance of $\xi(t)$ over the relevant interval is,

$$
2 \sum_{1}^{\infty}\left(A_{c n}^{2}+A_{s n}^{2}\right)=\frac{2}{T^{2}} \sum_{1}^{\infty}|\mathfrak{F}(i 2 \pi n / T)|^{2}=1
$$

where

$$
\mathscr{F}(i 2 \pi \nu)=\int_{0}^{T} \xi(t) e^{-i 2 \pi \nu t} d t
$$

is the Fourier transform of $\xi(t)$ computed over the interval $0 \leqslant t \leqslant T$.

Using the relations given in (2-12) and (2-13), the variable $\zeta(t)$ may be determined to be

$$
\zeta(t ; T)=\frac{T}{\pi} \sum_{1}^{\infty} \frac{1}{n}\left[A_{c n} \sin (2 n \pi t / T)-A_{s n} \cos (2 n \pi t / T)\right]
$$

The variance of $\zeta(t)$ will therefore be

$$
\begin{aligned}
\overline{\zeta^{2}}(t) & =\sigma_{\zeta}^{2}(T)=\frac{T^{2}}{2 \pi^{2}} \sum_{1}^{\infty} \frac{1}{n^{2}}\left(A_{c n}^{2}+A_{s n}^{2}\right) \\
& =\frac{1}{2 \pi^{2}} \sum_{1}^{\infty} \frac{1}{n^{2}}|\mathcal{F}(i 2 \pi n / T)|^{2} .
\end{aligned}
$$

Since the power spectral density of $\xi(t)$ is

$$
P_{\xi}(\nu)=\frac{2}{T}|\mathfrak{F}(i 2 \pi \nu)|^{2}
$$

the standard deviation of $\sigma_{\theta}$ may be readily determined from (3-8), (3-19), and (3-22). Thus,

$$
\begin{aligned}
\sigma_{\theta}(T) & =\epsilon T \sqrt{\frac{\sum_{1}^{\infty} \frac{1}{n^{2}} P_{\xi}\left(\frac{n}{T}\right)}{\sum_{1}^{\infty} P_{\xi}\left(\frac{n}{T}\right)}} \\
& =\epsilon \lambda(T)
\end{aligned}
$$

where $\lambda(T)$ is a factor that is dependent on only the spectral distribution of power in the motional instability function $\xi(t)$ and the analysis interval $T$ (correlator integration time).

Since the infinite summation involved in (3-24) may (in certain instances) be difficult to compute, a suitable approximation for $\lambda(T)$ may readily be shown to be

$$
\lambda(T) \approx \sqrt{\frac{\int_{1 / T}^{\infty} \frac{P_{\xi}(\nu)}{\nu^{2}} d \nu}{\int_{1 / T}^{\infty} P_{\xi}(\nu) d \nu} .}
$$


TABLE II

Dependence OF $\sigma_{\theta}(T)$ ON $q$

\begin{tabular}{|c||c|c|c|}
\hline$q$ & $Z(q)$ & $Z(q+2)$ & $\sigma_{\theta}(\mathrm{T})$ \\
\hline 1 & $\infty$ & 1.2021 & 0 \\
2 & 1.6449 & 1.0823 & $0.8112 \in \mathrm{T}$ \\
3 & 1.2021 & 1.0369 & $0.9287 \in \mathrm{T}$ \\
4 & 1.0823 & 1.0173 & $0.9695 \in \mathrm{T}$ \\
5 & 1.0369 & 1.0083 & $0.9858 \in \mathrm{T}$ \\
6 & 1.0173 & 1.0041 & $0.9934 \in \mathrm{T}$ \\
$\infty$ & 1 & 1 & $\epsilon \mathrm{T}$ \\
\hline
\end{tabular}

Using the results of (3-24) in (3-15) then, the correlation coefficient may be estimated from

$$
\cos (\epsilon \lambda) \leqslant \gamma_{N}(T) \leqslant e^{-(1 / 2) \epsilon^{2} \lambda^{2}} .
$$

\section{Example Power Spectral Distributions $P_{\xi}(\nu)$}

To illustrate the solution of the correlation degradation function using the relations derived in the previous analyses, several examples have been chosen to demonstrate the influence of the power spectral distribution of $\xi(t)$ on the correlation degradation. Of particular interest will be the dependence of the standard deviation $\sigma_{\theta}(T)$ on the correlation integration time $T$ for specific classes of power spectral density functions.

1) The Exponentially Decaying Power Spectrum: Consider first the case of an exponentially decaying power spectrum where the spectral power is concentrated at the low frequency extreme of $1 / T \mathrm{~Hz}$ and falls off exponentially with increasing frequency. Let the power spectral density function be represented as

$$
P_{\xi}(\nu)=(\nu T)^{-q} .
$$

This function is unity at $\nu=1 / T$ and falls off at $3 q \mathrm{~dB}$ per octave as the frequency is increased. Using (3-24), it is a simple matter to show that

$$
\sigma_{\theta}(T)=\epsilon T \quad \sqrt{\frac{Z(q+2)}{Z(q)}} \leqslant \epsilon T
$$

where $Z()$ is the Riemann Zeta function of the enclosed argument. For $q=1$, the above function is zero. As $q$ increases above one, the function increases monotonically and approaches $\epsilon T$ as $q$ goes to infinity. The dependence of the function $\sigma_{\theta}(T)$ on the parameter $q$ is illustrated in Table II.

2) The Uniform Low-Band Power Spectrum: Consider next a power spectrum which is essentially uniform over the frequency band from $1 / T \leqslant \nu \leqslant \nu_{0}$, after which the power spectrum falls off at an exponential rate. Let the power spectral density function be represented as

$$
P_{\xi}(\nu)=\frac{1}{1+\left(\nu / \nu_{0}\right)^{q}}, \quad(\text { for } 1 / T \leqslant \nu) .
$$

For $q$ greater than one this function is relatively constant out to the neighborhood of $\nu=\nu_{0}$, after which the power spectrum decays at a rate approaching $3 q \mathrm{~dB}$ octave at $\nu_{0}$. When the parameter $q$ is very large, the power spectrum takes the shape of a rectangle of unit height and width equal to $\nu_{0}-1 / T$. Thus,

$$
\lim _{q \rightarrow \infty} p_{\xi}(\nu)=h(\nu-1 / T)-h\left(\nu-\nu_{0}\right)
$$

where $h(x)$ is the Heaviside unit step function.

When $q=1$, the standard deviation $\sigma_{\theta}(T)$ will be zero as in the previous example. When $q=2$, the standard deviation becomes

$$
\begin{gathered}
\sigma_{\theta}(T)=\epsilon T \sqrt{\frac{\sum_{1}^{\infty} n^{-2}\left[1+\left(n / \nu_{0} T\right)^{2}\right]^{-1}}{\sum_{1}^{\infty}\left[1+\left(n / \nu_{0} T\right)^{2}\right]^{-1}}} \\
>0.72 \epsilon \sqrt{T / \nu_{0}}, \quad\left(\text { for } 1<\nu_{0} T\right) .
\end{gathered}
$$

As $q$ approaches infinity, the standard deviation approaches

$$
\begin{aligned}
\sigma_{\theta}(T) & =\epsilon \sqrt{\left(T / \nu_{0}\right) \sum_{1}^{v_{0} T} n^{-2}} \\
& <1.28 \in \sqrt{T / \nu_{0}}, \quad\left(\text { for } 1<\nu_{0} T\right) .
\end{aligned}
$$

3) The Peak Band-limited Power Spectrum: Consider finally a power spectrum which rises from an initial value (at $\nu=1 / T$ ) to some peak value at $\nu=\nu_{0}$, and then decays rapidly as $\nu$ increases beyond $\nu_{0}$. Let the power spectral density function be represented as

$$
P_{\xi}(\nu)=\left(\nu / \nu_{0}\right)^{q} e^{1-\left(\nu / \nu_{0}\right)^{q}} .
$$

The above function is maximum at $\nu=\nu_{0}$ and falls off on either side of this frequency. The peakedness of the function in the neighborhood of $\nu=\nu_{0}$ is a monotonically increasing function of the parameter $q$. As $q$ approaches infinity, the function approaches a singular point at $\nu=\nu_{0}$. Thus, for very large values of $q$, the power spectral density function can be represented as the Dirac delta function

$$
P_{\xi}(\nu)=\delta\left(\nu-\nu_{0}\right)
$$

When $q=2$, the standard deviation $\sigma_{\theta}(T)$ will be

$$
\begin{aligned}
& \sigma_{\theta}(T)= \sqrt{2} \frac{\epsilon}{\nu_{0}} \\
& \quad \sqrt{\frac{1-2 N\left(\sqrt{2} / \nu_{0} T\right)}{1-2 N\left(\sqrt{2} / \nu_{0} T\right)+\left(2 / \sqrt{\pi} \nu_{0} T\right) e^{-1 /\left(\nu_{0} T\right)^{2}}}} \\
&<\sqrt{2} \epsilon / \nu_{0} \quad\left(\text { for } 1<\nu_{0} T\right)
\end{aligned}
$$

where $N(\mathrm{)}$ is the integral of the Normal distribution function for the given argument.

As $q$ approaches infinity, the standard deviation approaches

$$
\sigma_{\theta}(T)=\epsilon / \nu_{0} .
$$

4) Summary Analysis: The results of the three classes of power spectral density functions previously analyzed are displayed in Table III for convenience.

The results demonstrate that a gross characterization of the power spectral density $P_{\xi}(\nu)$ is sufficient to permit a reasonably accurate prediction of the correlation degradation; particularly, when the resulting standard deviation $\sigma_{\theta}(T)$ is no greater than about one. (When $\sigma_{\theta}>1$, an accurate prediction of $\gamma_{N}$ is of little significance since the resulting correlation 
TABLE III

DEPENDENCE of $\sigma_{\theta}(T)$ AND $\gamma_{N}(T)$ ON $P_{\xi}(\nu)$

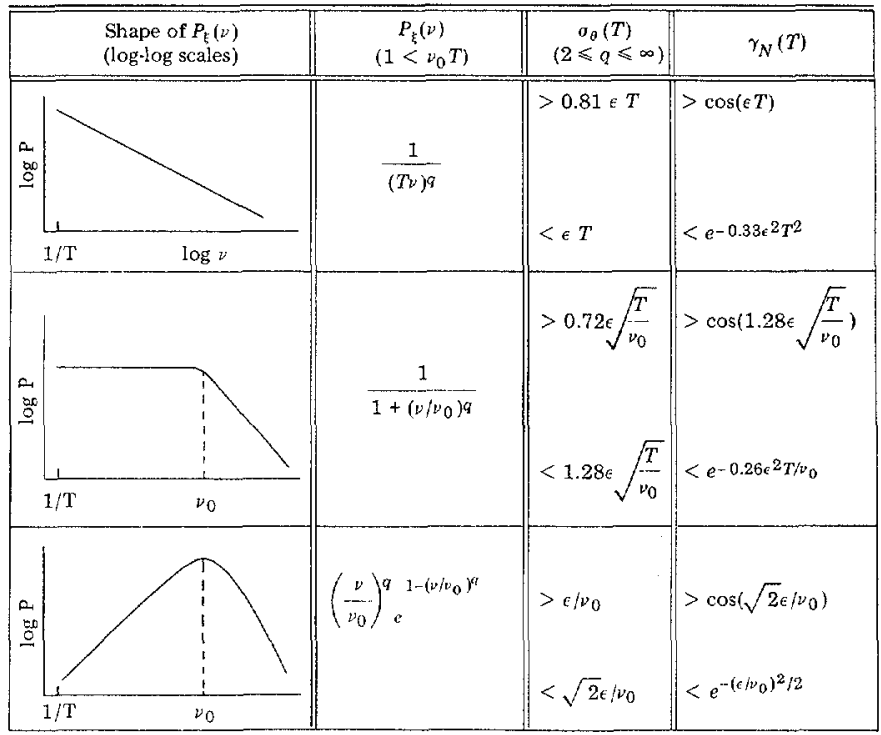

degradation has reached what can be considered intolerable levels.)

The analyses clearly indicate that the more the power spectral density of $\xi(t)$ is concentrated toward the low end of the frequency spectrum, the more severe will be the correlation degradation. Conversely, as the power spectral density becomes more concentrated at the higher frequencies, the less will be the correlation degradation. For power spectral distributions which fall off at a rate in excess of $3 \mathrm{~dB}$ per octave, the standard deviation $\sigma_{\theta}(T)$ will be directly proportional to the integration time $T$. For power spectral distributions which are relatively uniform out to some frequency $\nu_{0}$, after which they fall off at a rate in excess of 3 $\mathrm{dB}$ per octave of $\nu_{0}$, the standard deviation $\sigma_{\theta}(T)$ will be directly proportional to the square root of $T$ and inversely proportional to the square root of $\nu_{0}$. For power spectral distributions which rise at a rate equal to (or in excess of) $6 \mathrm{~dB}$ per octave out to some peak frequency $\nu_{0}$, after which they fall off at a rate in excess of $3 \mathrm{~dB}$ per octave, the standard deviation will be independent of the integration time $T$ and inversely proportional to the peak frequency $\nu_{0}$. In these latter two cases, it is assumed that the correlation integration time is greater than the inverse of the frequency $\nu_{0}$. (It should be apparent that as the integration time $T$ decreases and becomes less than the inverse critical frequency $\nu_{0}$, the latter two cases revert to the first case shown in Table III.)

It is reasonable to speculate that the nature of vehicle motional instability is more nearly represented by the third case shown in Table III. A transiting vehicle will generally undulate about some fixed course and speed as a pilot (or helmsman) attempts to maintain a prescribed speed and heading. It is reasonable to expect the frequency of the undulation to remain relatively constant over relatively long periods of time. Consequently, if the correlation integration time is appreciably greater than the inverse frequency of motional undulation, the resulting correlation degradation will be independent of the correlator integration time. In the next section, a particular example will be studied which demonstrates the correlation degradation as a function of the integration time for an idealized sinusoidal target motional variation.

\section{SPECIFIC SOLUTIONS OF THE CORRELATION Degradation Problem}

In the previous section, the general solution to the correlation degradation integral was discussed in some detail. In this section the degradation integral will be solved in closed form for specific target motional variables $\xi(t)$. The solutions will demonstrate a number of the principles covered in the earlier analyses.

\section{A. Sinusoidal Target Motion Variable}

For purposes of analysis, consider a target motion variable $\xi(t)$ which is a pure sinusoidal fluctuation with time, and whose frequency $\nu_{0}$ may be treated as a parameter. Over the interval $0 \leqslant t \leqslant T$, let the motional variable be represented as

$$
\begin{aligned}
\xi(t) & =A \sin \left(2 \pi \nu_{0} t-\phi\right) \\
& =A \sin \left(2 \frac{t}{T}-1\right) \phi
\end{aligned}
$$

where $\phi=\pi \nu_{0} T$ and where

$$
A= \pm \sqrt{\frac{2}{1-\sin (2 \phi) / 2 \phi}} .
$$

It can be verified that $\xi(t)$ is a zero mean variable over $0 \leqslant t \leqslant T$ with standard deviation equal to unity.

The selected target motion variable is a natural one, and one that is representative of a vehicle transiting a sinusoidal track with period $1 / \nu_{0}$. This is a natural choice to study, since any guided vehicle will follow a weaving path as the pilot (or control system) continually adjusts the vehicle heading to correct for base course deviations. When the parameter $\phi$ is made very small (viz. $\phi<<1$ ), the time scalefactor variable reduces to the simple linear function.

$$
\xi(t) \approx \pm \sqrt{3}\left(2 \frac{t}{T}-1\right) .
$$

This is representative of linear change in speed (or a linear change in course) over the analysis interval $T$.

Applying (2-12) and (2-13), the variable $\zeta(t)$ is computed to be

$$
\zeta(t)=\frac{A T}{2 \phi}\left[\frac{\sin \phi}{\phi}-\cos \left(1-2 \frac{t}{T}\right) \phi\right]
$$

and

$$
\overline{\zeta^{2}}(t)=\left(\frac{T}{2 \phi}\right)^{2} \frac{1+\sin (2 \phi) / 2 \phi-2 \sin ^{2}(\phi) / \phi^{2}}{1-\sin (2 \phi) / 2 \phi} .
$$

As $\phi$ gets small $(\phi<<1)$ the above functions approach

$$
\zeta(t)=\frac{T}{4 \sqrt{3}}\left[3(1-2 t / T)^{2}-1\right]
$$

and

$\overline{\zeta^{2}}=T^{2} / 60$. 


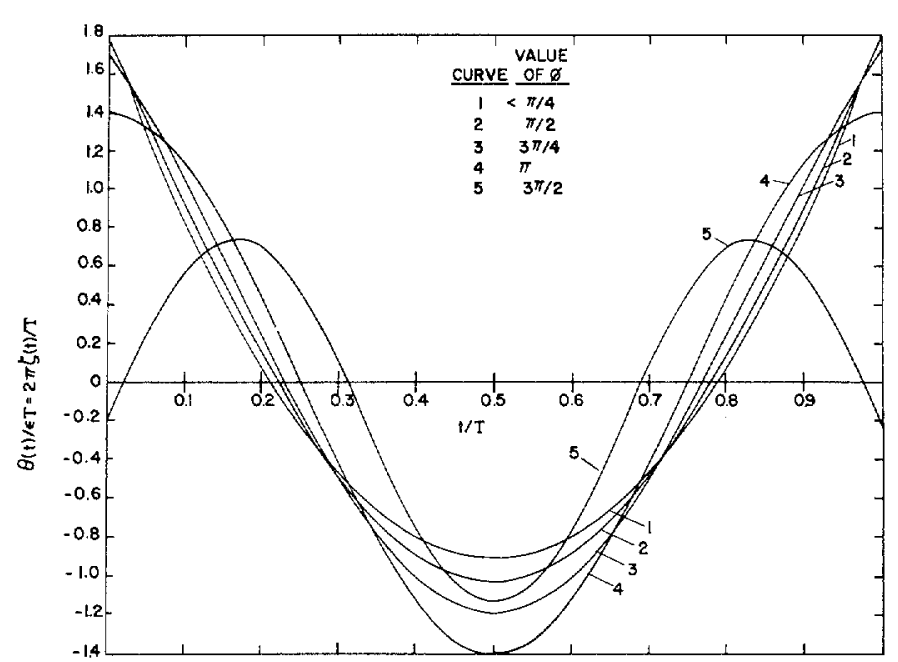

Fig. 3. Plots of $\theta(t) / \epsilon T$ for selected values of $\phi$.

Using (3-7), plots of $\theta(t) / \epsilon T$ have been computed for selected values of $\phi$. The results are shown in Fig. 3. The curves illustrate the minimal interchannel phase-difference error resulting from the target motional instability. The function reaches its lower limiting bound (Curve 1) for $\phi$ approximately equal to $\pi / 4$. At the higher values of $\phi$, the phase curves will be sinusoidal in form.

\section{B. Correlation Degradation}

In the present example, the correlation degradation may be solved for directly by using the first integral representation in (3-9). The solution becomes

$$
\begin{aligned}
\gamma_{N}(T)= & \frac{1}{\phi} \int_{0}^{\phi} \cos \left\{\frac{\epsilon T}{b(\phi)}[a(\phi)-\cos x]\right\} d x \\
= & \cos \left[\frac{a(\phi)}{b(\phi)} \epsilon T\right] \frac{1}{\phi} \int_{0}^{\phi} \cos \left[\frac{\epsilon T}{b(\phi)} \cos x\right] d x \\
& +\sin \left[\frac{a(\phi)}{b(\phi)} \epsilon T\right] \frac{1}{\phi} \int_{0}^{\phi} \sin \left[\frac{\epsilon T}{b(\phi)} \cos x\right] d x
\end{aligned}
$$

where

$$
a(\phi)=\sin (\phi) / \phi
$$

and

$$
b(\phi)=\frac{\phi}{\pi} \sqrt{\frac{1}{2}[1-\sin (2 \phi) / 2 \phi]} .
$$

The two integral functions in (4-8) are closely related to the Bessel functions. In the particular case where $\phi=$ $m \pi\left(\nu_{0} T=m\right),(4-8)$ reduces to

$$
\begin{aligned}
\gamma_{N}(T) & =J_{0}(\sqrt{2} \epsilon T / m) \\
& =J_{0}\left(\sqrt{2} \epsilon / \nu_{0}\right)
\end{aligned}
$$

On the other hand, when $\phi<\pi / 4 \quad\left(\nu_{0} T<1 / 4\right)$, the correlation degradation approaches

$$
\begin{aligned}
\gamma_{N}(T)= & \frac{\cos \left(\frac{\pi \epsilon T}{2 \sqrt{3}}\right)}{\sqrt{\sqrt{3} \epsilon T}} \int_{0}^{\sqrt{\sqrt{3} \epsilon T}} \cos \left(\frac{\pi}{2} x^{2}\right) d x \\
& +\frac{\sin \left(\frac{\pi \epsilon T}{2 \sqrt{3}}\right)}{\sqrt{\sqrt{3} \epsilon T}} \int_{0}^{\sqrt{\sqrt{3} \epsilon T}} \sin \left(\frac{\pi}{2} x^{2}\right) d x \\
= & \frac{1}{\sqrt{\sqrt{3} \epsilon T}}\left[\cos \left(\frac{\pi \epsilon T}{2 \sqrt{3}}\right) C(\sqrt{\sqrt{3} \epsilon T})\right. \\
& \left.+\sin \left(\frac{\pi \epsilon T}{2 \sqrt{3}}\right) S(\sqrt{\sqrt{3} \epsilon T})\right]
\end{aligned}
$$

where $S()$ and $C()$ are the Fresnel sine and cosine integrals for the indicated argument.

Plots of the correlation degradation for selected values of $\phi$ are illustrated in Fig. 4 . It will be observed that when the parameter $\phi$ is greater than $\pi$, the correlation degradation decreases as $\phi$ is increased. On the other hand, the correlation also decreases for decreasing values of $\phi$ below $\pi$. The limiting value of the function is reached for $\phi$ approximately equal to $\pi / 4$. Thus, the lower bound on the correlation degradation function is achieved when $\phi=\pi$. This fact could have been predicted from the earlier analysis based upon power spectral distribution considerations. It will be recalled that the more the power in $\xi(t)$ becomes concentrated at the lower frequency $1 / T$, the larger will be the value of the standard deviation $\sigma_{\theta}(T)$. Consequently, as $\phi=\pi \nu_{0} T$ is lowered to the value $\pi$, the power spectral density of $\xi(t)$ becomes maximally concentrated at the frequency $1 / T$. As $\phi$ is further decreased below the value $\pi$, the spectral power of $\xi(t)$ is partitioned into the fundamental frequency $1 / T$ and the harmonics of the fundamental frequency. Thus, the spectral power of $\xi(t)$ is spread over a wide range of frequencies and $\sigma_{\theta}(T)$ decreases from its maximum value. This phenomenon will become evident in the power spectrum analysis to follow.

\section{Power Spectrum $P_{\xi}(\nu)$ and Standard Deviation $\sigma_{\theta}(T)$}

It will prove informative to demonstrate the standard deviation $\sigma_{\theta}(T)$ approach to the correlation degradation for the specific example selected, since this approach has more universal application.

From (3-20), (3-23), and (4-1), the power spectral density of $\xi(t)$ may be computed as

$$
P_{\xi}(\nu)=\frac{A^{2} T}{2}\left[\frac{\sin \pi T\left(\nu-\nu_{0}\right)}{\pi T\left(\nu-\nu_{0}\right)}-\frac{\sin \pi T\left(\nu+\nu_{0}\right)}{\pi T\left(\nu+\nu_{0}\right)}\right]^{2}
$$

and

$$
P_{\xi}\left(\frac{n}{T}\right)=\frac{2}{\pi^{2}} A^{2} T \sin ^{2} \pi \nu_{0} T \frac{n^{2}}{\left[n^{2}-\left(\nu_{0} T\right)^{2}\right]^{2}}
$$

when $\nu_{0} T \neq m \quad(\phi \neq m \pi)$. For $\nu_{0} T=m \quad(\phi=m \pi)$

$$
P_{\xi}\left(\frac{n}{m} \nu_{0}\right)=\left\{\begin{array}{ll}
0 & (\text { for } n \neq m) \\
T & (\text { for } n=m)
\end{array}\right\} .
$$




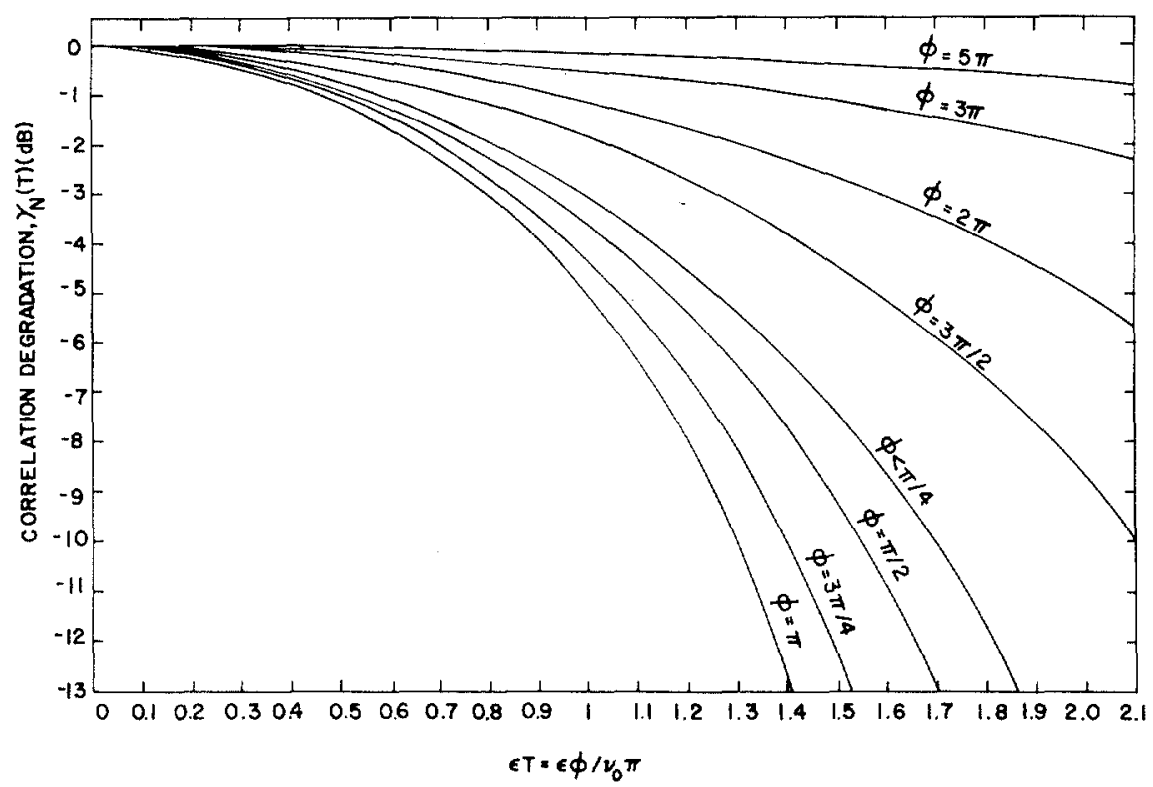

Fig. 4. Plots of $\gamma_{N}(T)$ as a function of $T$.

For $\nu_{0} T<1 / 4 \quad(\phi<\pi / 4)$, the power spectral density approaches

$$
p\left(\frac{n}{T}\right)=6 T / \pi^{2} n^{2}
$$

Using (3-8), (3-24), and (4-5), the standard deviation may be computed as

$$
\begin{aligned}
\sigma_{\theta}\left(T ; \nu_{0}\right) & =\epsilon T \sqrt{\frac{\sum_{1}^{\infty}\left[n^{2}-\left(\nu_{0} T\right)^{2}\right]^{-2}}{\sum_{1}^{\infty} n^{2}\left[n^{2}-\left(\nu_{0} T\right)^{2}\right]^{-2}}} \\
& =\frac{\epsilon}{\nu_{0}} \sqrt{\frac{1+\sin (2 \phi) / 2 \phi-2 \sin ^{2}(\phi) / \phi^{2}}{1-\sin (2 \phi) / 2 \phi}} .
\end{aligned}
$$

For $\nu_{0} T=m(\phi=m \pi)$, the standard deviation is simply $\epsilon / \nu_{0}=\epsilon T / m$. And for $\nu_{0} T<1 / 4(\phi<\pi / 4)$, the standard deviation of $\theta$ approaches

$$
\sigma_{\theta}(T)=\epsilon T \sqrt{Z(4) / Z(2)}=\pi \epsilon T / \sqrt{15} \approx 0.81 \epsilon T .
$$

A plot of $\sigma_{\theta}(\theta)$ as a function of $\nu_{0} T$ (or $\left.\phi / \pi\right)$ is illustrated in Fig. 5. Two curves are shown to delineate the influence of both the integration time $T$, and the frequency $\nu_{0}$ on the standard deviation $\sigma_{\theta}$.

A study of Fig. 5 demonstrates a number of the properties inherent in the sinusoidal target motion variable. It will be noticed that for $\nu_{0}$ fixed and $\nu_{0} T<1 / 4$, the value of $\sigma_{\theta}(T)$ will be proportional to the correlator integration time $T$. This was predictable since the power spectral density of $\xi(t)$ falls off at a rate inversely proportional to the square of the frequency (see Table III). The correlation degradation increases with increased integration time up to the point where $T$ becomes greater than $1 / \nu_{0}$. After this point, the correlation degradation levels off (oscillating slightly about some fixed value) as $T$ is further increased. When $T$ is much greater than $1 / \nu_{0}$, the value of $\sigma_{\theta}(T)$ will be (essentially) independent of the integration time $T$. This was also predictable since the power spectral density of $\xi(t)$, given in (4-11) or (4-12), fits the general characteristics of the third curve in Table III.

For a fixed integration time, the dependence of $\sigma_{\theta}\left(T ; \nu_{0}\right)$ on the frequency $\nu_{0}$ is given by the broken curve (Fig. 5). This curve shows that $\sigma_{\theta}$ maximizes for $\nu_{0}=1 / T$ as discussed earlier. At this frequency the spectral power in $\xi(t)$ is maximally concentrated at the frequency $1 / T$.

The above example clearly demonstrates the applicability of the power spectral density approach to determine the standard deviation of the phase function $\theta(t)$. The merit of using $\sigma_{\theta}$ to predict the correlation degradation should be evident from the earlier analyses.

\section{Target Motion Step Function}

As a second specific example, consider that the target motion variable $\xi(t)$ is a step function, where the step occurs somewhere in the interval $0 \leqslant t \leqslant T$. That is, let

$$
\xi(t)=\left\{\begin{aligned}
-\sqrt{(1-\mu) / \mu} & (\text { for } 0 \leqslant t \leqslant \mu T \\
\sqrt{\mu /(1-\mu)} & \text { (for } \mu T \leqslant t \leqslant T)
\end{aligned}\right\} .
$$

(It can be readily verified that $\xi$ is a zero mean variable with unity variance.) Such a function represents a fixed change in the target speed (or course) in the situation where the time required to effect the change is small compared with the integration time $T$. In this situation, $\sigma_{v}$ in (2-8) or $\sigma_{\varphi}$ in (2-9) is simply equal to

$$
\sigma_{v}=\sqrt{\mu(1-\mu)} \Delta v
$$

or

$$
\sigma_{\varphi}=\sqrt{\mu(1-\mu)} \Delta \varphi
$$

where $\Delta v$ and $\Delta \varphi$ represent the step changes in speed and course, respectively.

Following the procedures instituted in the earlier analyses, it can be verified that 


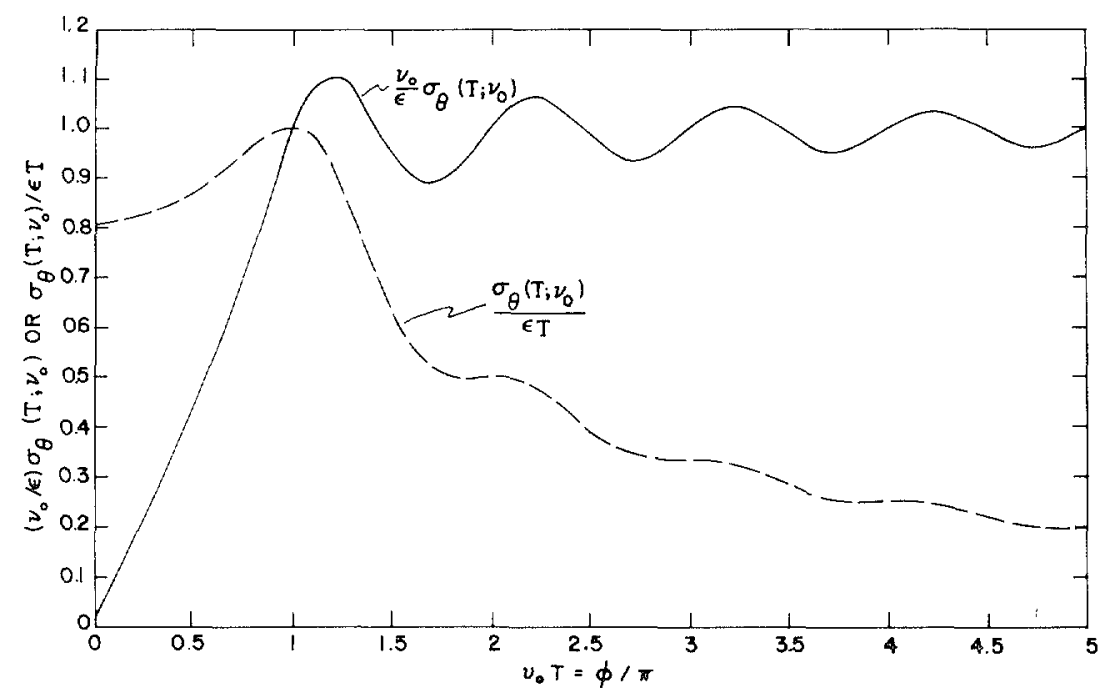

Fig. 5. Plot of $\sigma_{\theta}(T)$ as a function of $T$ and $\nu_{0}$.

$$
\zeta(t)=\left\{\begin{array}{lr}
\sqrt{(1-\mu) / \mu}(\mu T / 2-t) & (0 \leqslant t \leqslant \mu T) \\
\sqrt{\mu /(1-\mu)}[t-(1+\mu) T / 2] & (\mu T \leqslant t \leqslant T)
\end{array}\right\}
$$

and

$$
\begin{aligned}
\sigma_{\theta}(T ; \mu) & =\pi \sqrt{\mu(1-\mu) / 3} \epsilon T \\
& =(\pi / \sqrt{3}) \mu(1-\mu) \epsilon^{\prime} T
\end{aligned}
$$

where $\epsilon^{\prime}=\epsilon / \sqrt{\mu(1-\mu)}$. The parameter $\epsilon^{\prime}$ is the significant parameter (in this case) since, from (4-18) and (4-19), a direct substitution of the magnitude of the step change can be made for the standard deviation in the parameter $\epsilon$.

The maximum value of $\sigma_{\theta}(T ; \mu)$ occurs for $\mu=0.5$. Thus,

$$
\sigma_{\theta \max }(T)=\frac{\pi}{4 \sqrt{3}} \epsilon^{\prime} T \approx 0.45 \epsilon^{\prime} T \text {. }
$$

Since $\mu$ may take on values from 0 to 1 with equal probability, the mean or expected value of the standard deviation will be

$$
\begin{aligned}
\sigma_{\theta E}(T) & =\frac{\pi}{\sqrt{3}} \epsilon^{\prime} T \int_{0}^{1} \mu(1-\mu) d \mu \\
& =\frac{\pi}{6 \sqrt{3}} \epsilon^{\prime} T=\frac{2}{3} \sigma_{\theta \max }(T) .
\end{aligned}
$$

From (4-20) it is evident that $\theta$ will be uniformly distributed over the range of $\theta= \pm \mu(1-\mu) \pi \epsilon^{\prime} T$. Consequently,

$$
\gamma_{N}(T ; \mu)=\sin \left[\mu(1-\mu) \pi \epsilon^{\prime} T\right] / \mu(1-\mu) \pi \epsilon^{\prime} T .
$$

The expected value of the correlation coefficient is therefore,

$$
\gamma_{E}(T)=\frac{\sin \left(\pi \epsilon^{\prime} T / 6\right)}{\pi \epsilon^{\prime} T / 6}
$$

\section{E. Variable Geometry Problem}

In the earlier analyses, the variation of the system geometry with target motion has been assumed to be negligible. The basic problem had been formulated on the assumption that only the target course and speed were variable. It will now be well to study the correlation degradation due to variations in the system geometry resulting from the target motion. For this analysis, the target course and speed will be assumed to be constant.

From (2-6) and (2-7) it can readily be shown that the time scale-factor shift due to the variation in system geometry may be approximated as

$$
\begin{aligned}
\delta_{y x} \approx & \left\langle2 \frac { v _ { 0 } } { c } \left\{\sin \frac{1}{2} \alpha \sin \varphi_{0}\right.\right. \\
& +\frac{v_{0}}{7200}\left[\frac{\sin ^{2}\left(\beta-\beta_{0 x}\right)}{R_{x}}-\frac{\sin ^{2}\left(\beta-\beta_{0 y}\right)}{R_{y}}\right](t-T / 2) \\
& +\left(\frac{v_{0}}{7200}\right)^{2} \cos \frac{1}{2} \alpha \cos \varphi_{0}\left[\frac{\sin ^{2}\left(\beta-\beta_{0 x}\right)}{R_{x}^{2}}\right. \\
& \left.\left.\left.-\frac{\sin ^{2}\left(\beta-\beta_{0 y}\right)}{R_{y}^{2}}\right](t-T / 2)^{2}\right\}\right\rangle_{t, a}
\end{aligned}
$$

when $v_{0} T<<R_{x}$ or $R_{y}$. (That is, when the distance traveled by the target over the analysis interval $T$ is much smaller than the distance from the target to both of the two receiving sensors.) In the preceding equation, the velocity units are in knots, the range units are in nautical miles and the units of time are in seconds.

If now, the target speed $v_{0}$ and the target course $\beta$ are assumed constant, the time scale-factor shift can be reduced to

$$
\begin{aligned}
\delta_{y x} \approx & 2\left(v_{0} / c\right) \sin \frac{1}{2} \alpha \sin \varphi_{0} \\
& +\frac{v_{0}^{2} T}{7200 \sqrt{3} c}\left[\frac{\sin ^{2}\left(\beta-\beta_{0 x}\right)}{R_{x}}\right. \\
& \left.-\frac{\sin ^{2}\left(\beta-\beta_{0 y}\right)}{R_{y}}\right]\langle\xi(t)\rangle_{t, \alpha}
\end{aligned}
$$

where

$$
\xi(t)=\sqrt{3}[2(t / T)-1] .
$$

(The third term in (4-26) has been dropped since, in general, this term is much smaller than the second term and will contribute negligibly to the correlation degradation.)

It will be noted that $\xi(t)$ (in this case) is identical to that 
given in (4-3). Consequently, the solution for $\zeta(t)$ will be that given by (4-6).

For convenience, a parameter $\epsilon_{0}$ will be defined as

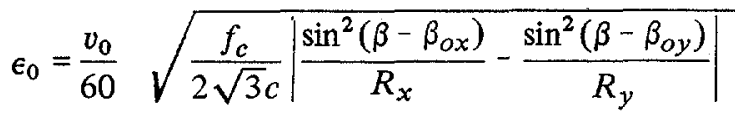

where

$$
\begin{array}{ll}
v_{0} & \text { is the target speed in knots; } \\
f_{c} & \text { is the center frequency of the target source in } \mathrm{Hz} ; \\
c & \text { is the mean propagation velocity in knots; } \\
R_{x}, R_{y} & \text { are the ranges of the target to the respective re- } \\
\beta & \text { ceiving sensors in nautical miles; } \\
\beta_{o x}, \beta_{o y} & \text { is the angle of the target course (see Fig. 1); } \\
& \text { target to the respective receiving sensors (see } \\
\text { Fig. 1). }
\end{array}
$$

Following the procedures instituted in the earlier analyses, it is a simple matter to show that

$$
\begin{aligned}
& \theta(t)=\frac{\pi}{2 \sqrt{3}}\left(\epsilon_{0} T\right)^{2}\left[3\left(2 \frac{t}{T}-1\right)^{2}-1\right] \\
& \sigma_{\theta}(T)=\frac{\pi}{\sqrt{15}}\left(\epsilon_{0} T\right)^{2} \approx 0.81\left(\epsilon_{0} T\right)^{2}
\end{aligned}
$$

and

$$
\begin{gathered}
\gamma_{N}(T) \frac{1}{\sqrt[4]{3} \epsilon_{0} T}\left\{\cos \left[\frac{\pi\left(\epsilon_{0} T\right)^{2}}{2 \sqrt{3}}\right] C\left(\sqrt[4]{3} \epsilon_{0} T\right)\right. \\
\left.+\sin \left[\frac{\pi\left(\epsilon_{0} T\right)^{2}}{2 \sqrt{3}}\right] S\left(\sqrt[4]{3} \epsilon_{0} T\right)\right\}
\end{gathered}
$$

where $S()$ and $C()$ are the Fresnel sine and cosine integrals for the indicated argument.

It will be noted that (4-31) and (4-32) have the same form as (4-16) and (4-10) with $\epsilon T$ being replaced by $\left(\epsilon_{0} T\right)^{2}$. A plot of the resulting correlation degradation as a function of $\epsilon_{0} T$ is given in Fig. 6. It will be noted that the correlation degradation increases rapidly for values of $\epsilon_{0} T$ greater than about 0.9 .

In any practical problem, the combination of the variable geometry (due to the mean target track) and target motional anomalies will both be present to cause phase correlation degradation. To obtain an estimate of the combined effect on the correlation degradation, one need only incoherently sum the phase standard deviations for each of the individual contributors. Thus,

$$
\sigma_{\theta}(T)=\sqrt{\sigma_{\theta 1}^{2}(T)+\sigma_{\theta 2}^{2}(T)}
$$

The resulting standard deviation for the phase error may then be used with (3-15) or with Fig. 2 to obtain an estimate of the correlation degradation for the combined distortion inducing mechanisms.

\section{Optimizing the Correlator INTEgRation Time}

With the solution of the correlation degradation integral, the question arises as to what integration time would be suitable or optimum when correlation processing is to be employed in a practical problem. Since degradation is never a desirable result, one would be motivated to keep the integration time

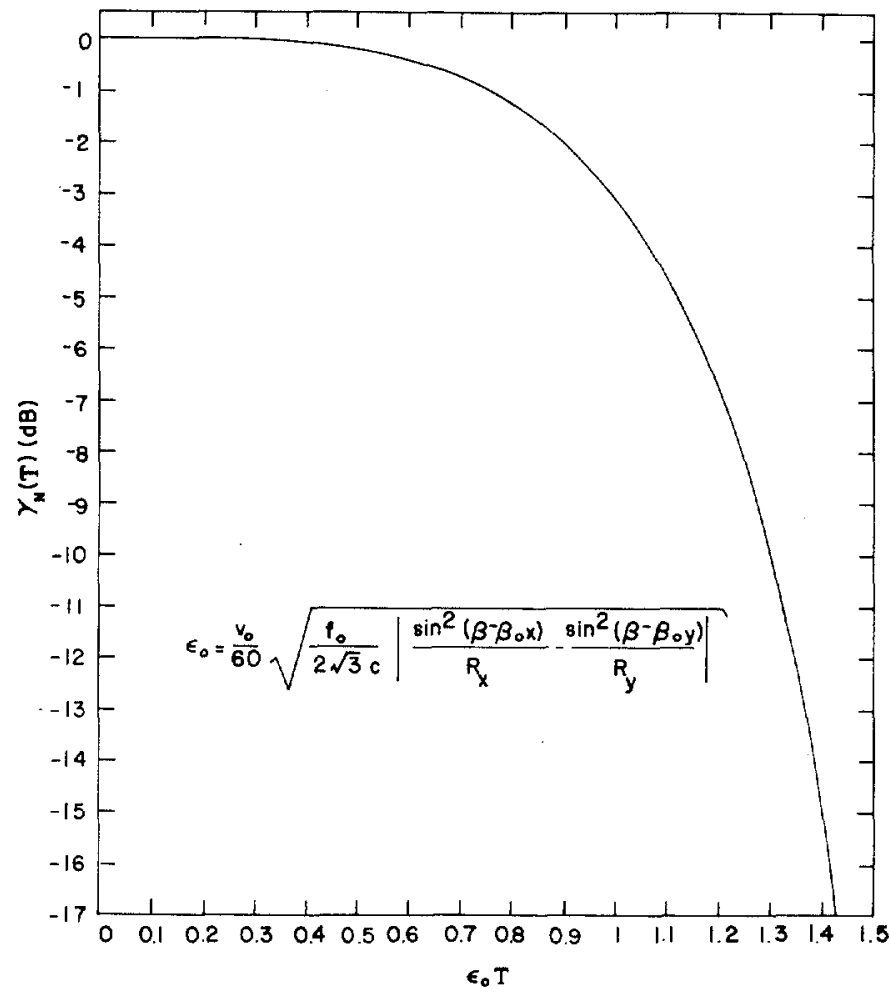

Fig. 6. Plot of $\gamma_{N}(T)$ as a function of $\epsilon_{0} T$.

small to minimize the correlation degradation due to target motion anomalies. On the other hand, the use of a large integration time is desirable from the standpoint of detection sensitivity and motional parameter resolution. Since detection is probably the most critical factor in most practical applications of correlation processors, the maximization of signal processor gain is the one which will be addressed in this report.

\section{A. Maximizing the Signal Processing Gain}

It is well known that the processing gain of a signal cross correlator is directly proportional to the square root of the correlator integration time. ${ }^{3}$ Consequently, there is ample motivation to increase the integration time of the correlation processor to the point where any further improvement in processing gain (achieved by increased integration time) is offset by the degradation due to the target motional effects. Based upon this philosophy, it would appear desirable to maximize the processor gain function

$$
G_{1}(T)=c_{1} T^{1 / 2} \gamma_{N}(T)
$$

where $c_{1}$ is a proportionality constant.

On the other hand, detection enhancement can also be achieved through postcorrelation integration (nonphase coherent) processing of the correlation processor output. Though not as sensitive, postcorrelation summation is also time dependent. The gain achieved through postcorrelation integration of the correlator output will generally be proportional to the fourth root of the ratio of the total processing time to the correlator (or coherent) integration time. For a total processing time (coherent and incoherent) of $T_{1} \mathrm{~s}$, then,

${ }^{3}$ See for example, A. A. Gerlach, "Theory and Applications of Statistical Wave-Period Processing," Vol. I, Gordon \& Breach Science Publishers Inc., Ch. 3, p. 127, (3.11-31); 1970. 
the overall processing gain would be

$$
G_{2}(T)=c_{2}\left(T_{1} / T\right)^{1 / 4} T^{1 / 2} \gamma_{N}(T)=c_{2}\left(T_{1} T\right)^{1 / 4} \gamma_{N}(T) .
$$

In this situation, optimum results will be achieved by choosing the integration time $T$ which maximizes (5-2).

In the interests of generality, let the processor gain function be

$$
G(T)=c_{0} T^{q} \gamma_{N}(T)
$$

where the decision on a suitable choice for $q$ can be deferred until the time of application. The procedure will therefore be to find the value of $T$ which maximizes (5-3). A suitable approach is to solve the equation

$$
\frac{d}{d T} G(T)=0
$$

for $T$. Carrying out the indicated operations given,

$$
\begin{aligned}
& \frac{d \gamma_{N}}{d T}+\frac{q}{T} \gamma_{N} \\
& =\frac{d \sigma_{\theta}}{d T} \frac{d \gamma_{N}}{d \sigma_{\theta}}+\frac{q}{T} \gamma_{N}=0 .
\end{aligned}
$$

Since $\gamma_{N}$ is a monotonically decreasing function of $\sigma_{\theta}$, and since the form of $\sigma_{\theta}(T)$ is known to approximate (over, at least, a portion of the range of $T$ )

$$
\sigma_{\theta}(T)=a T^{p}
$$

it will be convenient to solve (5-5) in terms of the phase standard deviation $\sigma_{\theta}(T)$.

From (5-5) and (5-6) then,

$$
\frac{d \gamma_{N}}{d \sigma_{\theta}}+\frac{q}{p} \frac{\gamma_{N}}{\sigma_{\theta}}=0
$$

Using the functional bounds on $\gamma_{N}$ given in (3-15) the solutions to (5-7) are readily determined to be

$$
\sigma_{\theta}=\sqrt{q / p}
$$

and

$$
\sigma_{\theta} \tan \sigma_{\theta}=q / p \text {. }
$$

A plot of the above bounds on $\sigma_{\theta}(T)$ as a function of the ratio $q / p$ is shown in Fig. 7. For representative choices of $q$ and $p$, the optimum choice for $\sigma_{\theta}(T)$ will range from 0.35 to about 0.8 .

It should be noted that the preceding procedure for determining an optimum $\sigma_{\theta}$ is predicated on the relation given in $(5-6)$. Since this relationship may be valid for only a limited region of the parameter $T$, the resulting processing gain (thus derived) may be only a localized maximum. One is therefore cautioned to examine the behavior of $\sigma_{\theta}(T)$ over an extended range of values of $T$ to see whether the localized maximum is equaled or exceeded at larger values of integration time.

\section{B. Sensitivity of the Processing Gain to Integration Time}

The purpose of the present analysis is to find the optimum integration time for a correlation processor from the standpoint of maximizing the overall detection processing gain.

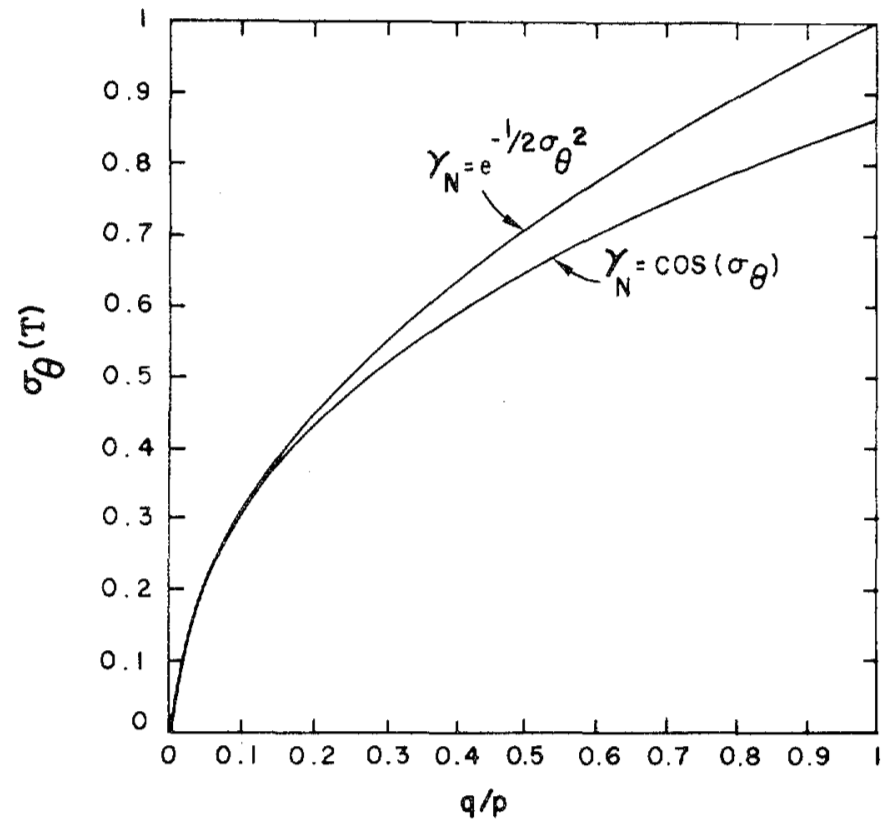

Fig. 7. Optimum standard deviation $\sigma_{\theta}(T)$ as a function of the ratio $q / p$.

Although the procedures for accomplishing this objective have been developed, the true value of these procedures will be dependent on the sensitivity of the processing gain to the correlation integration time. The reason for this is that the optimum integration time is dependent on a number of uncontrollable parameters (target location, speed, course, motional behavior, etc.) which are neither known precisely nor stationary with time. Consequently, the determination of the optimum integration time will (in many situations) be a best estimate, based upon limited knowledge about the relevant parameters involved in the optimization equation. Therefore, it will be worthwhile to study the sensitivity of the processing gain as a function of the correlator integration time to determine the effect of imprecisions in the selection of this processing parameter.

From (5-3) the signal processing gain can be normalized to unity for the optimum choice of integration time. Thus,

$$
G_{N}(T)=\left(T / T_{0}\right)^{q} \gamma_{N}(T) / \gamma_{N}\left(T_{0}\right)
$$

where $T_{0}$ is the optimum integration time for the correlator processor. (For a given standard deviation function $\sigma_{\theta}(T)$, the value of $T_{0}$ may be determined using the relations derived in the previous analysis.)

For purposes of the gain sensitivity study, two of the earlier examples have been chosen where the standard deviation $\sigma_{\theta}(T)$ is first directly proportional to $T$ and then to $T^{2} \quad(p=1$ and 2). Equation (5-9) has been computed in each case for values of $q$ equal to $\frac{1}{4}$ and $\frac{1}{2}$. The results are shown in Fig. 8 . The upper graph [Fig. 8(a)] illustrates the case where $\sigma_{\theta}(T)$ is directly proportional to the integration time, and the lower graph [Fig. 8(b)] illustrates the case where $\sigma_{\theta}(T)$ is proportional to the square of the integration time. From these curves it is evident that the processing gain is more highly sensitive to the integration time when $\sigma_{\theta}(T)$ is proportional to the square of $T$. The sensitivity is also greater for the higher value of $q$ (when $p$ is held constant). In any case, the processor gain sensitivity is sufficiently low so that a reasonable error in the 


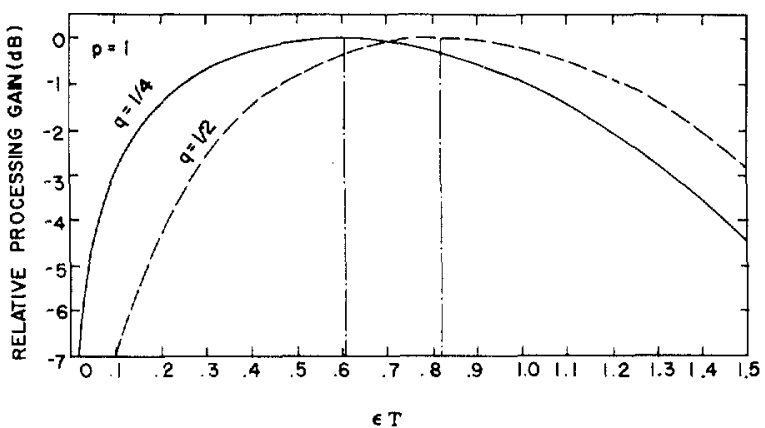

(a)

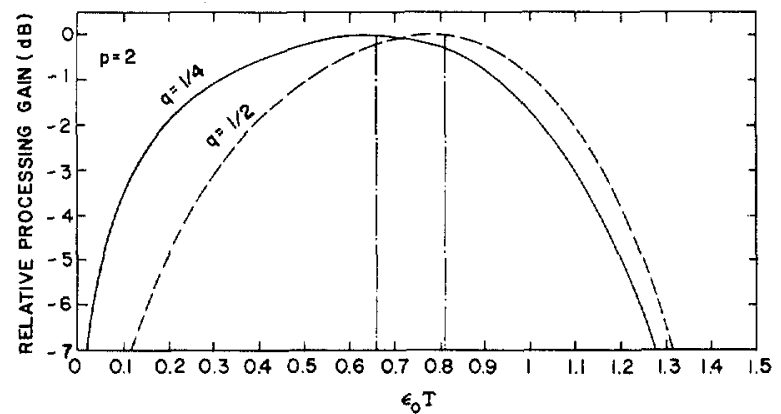

(b)

Fig. 8. (a) Standard deviation $\sigma_{\theta}(T)$ proportional to integration time: $\sigma_{\theta}(T)=0.81 \epsilon T$. (b) Standard deviation proportional to square of integration time: $\sigma_{\theta}(T)=0.81\left(\epsilon_{0} T\right)^{2}$.

estimate of $\epsilon$ (or $\epsilon_{0}$ ) can be made without suffering an intolerable loss in system processing gain. For a $1 \mathrm{~dB}$ loss tolerance in processing gain, the variation in $\epsilon T$ can range from about \pm 44 percent $\left(q=\frac{1}{2}\right)$ to about \pm 60 percent $\left(q=\frac{1}{4}\right)$, in the case where $\sigma_{\theta}(T)$ is proportional to $T$. For the other case, the variation in $\epsilon_{0} T$ can range from about \pm 34 percent $\left(q=\frac{1}{2}\right.$ ) to about 49 percent $\left(q=\frac{1}{4}\right)$, under the same loss restriction.

\section{Conclusions}

Correlation signal processing has proven its value in a number of practical applications involving motional target vehicles. Although generally successful over temporal limits of integration which are relatively short, little was known about the maximum integration to which coherent signal processing could be usefully applied. The subject paper addressed this problem from the standpoint of the correlation degradation experienced as a result of the target motion. The expected correlation degradation was determined to be a complex function of 1) the correlator integration time, 2) the mean frequency of the source signal, 3) the source-sensor system geometry, and 4) the nature and characteristics of the target motion.

Two important aspects of the target motion were found significant. These were the mean target track relative to the source-sensor geometry, and the target dynamic instabilities relative to the mean track. The mean target track induces correlation degradation as a result of the systematic changes in the source-sensor geometry over time. This degradation increases severely with correlator integration time for the higher value of integration time (see Fig. 6). This phenomenon creates an absolute upper bound on the useful coherence integration time which can be employed in any physical system.
Regarding the target dynamic instabilities, the induced correlation degradation was found to be dependent on the standard deviations of the target course and/or speed, and on the spectral distribution of these dynamic deviations. The correlator degradation is most severe when the spectral power (of the dynamic deviations) is highly concentrated at the lower frequency of $1 / T \mathrm{~Hz}$ (where $T$ is the correlator integration time). Conversely, the correlator degradation becomes less severe as the spectral power becomes more highly concentrated at the upper frequencies (see Table III and Figs. 4 and 5). When the spectral power has a lower bound limit of $\nu_{0} \mathrm{~Hz}$, the correlation degradation will become essentially bounded (independent of the correlation integration time) as $T$ becomes greater than about $1 / \nu_{0} \mathrm{~s}$ (see Table III and Fig. 5).

The selection of an optimum integration time for a correlation processor was investigated from the standpoint of maximizing the processor gain for signal detection in an incoherent signal background. Examples of the optimum relations are plotted in Fig. 8. The sensitivity of the optimum relations is sufficiently low so that a reasonable error in the estimate of the system parameters can be made without suffering an intolerable loss in system processing gain.

In summary, it can be concluded that target motion poses a fundamental limitation on the useful integration time for correlation processors.

\section{APPENDIX}

\section{Motion Induced Time SCAle-Factor Distortion}

When a signal source is in motion in a transmission media, the ideal signal received at a remote sensor in the medium will be a distorted replica of the source signal. The received signal, in this circumstance, can be represented as the original signal whose time scale is transformed (or distorted) in a manner which is functionally dependent on the source motional dynamics.

Fig. A-1 depicts the general system geometry under consideration. The source $o$ is proceeding along a target track while transmitting a signal $\mu(t)$. An observer, at position $x$, will receive a signal $\mu_{x}(t)$ which is dependent on both the signal $\mu(t)$ and the target motional dynamics. The problem being addressed is to determine the signal $\mu_{x}(t)$ and to show that, if we ignore signal attenuation and translation (time delay), the received signal can be written simply as $u(k t)$, where the time scale-factor $k$ can be defined in terms of the system geometry and the motional dynamics of the source.

\section{Differential Time Approach}

Consider first the differential time approach. Aboard the moving vehicle, the source signal will change from $u(t)$ to $u(t+d t)$ over the differential time $d t$. At the receiving sensor $x$, the time differential for the same change in the received signal (ignoring attenuation and time delays) will generally be compressed or expanded due to the component of source motion along the transmission path. Thus, the differential time $d t$ will be transformed into a differential time $d(k t)$. The time scale-factor $k$ will be functionally dependent on the component of velocity $v_{x}$ (see Fig. A-1). From the figure, it is evident that 


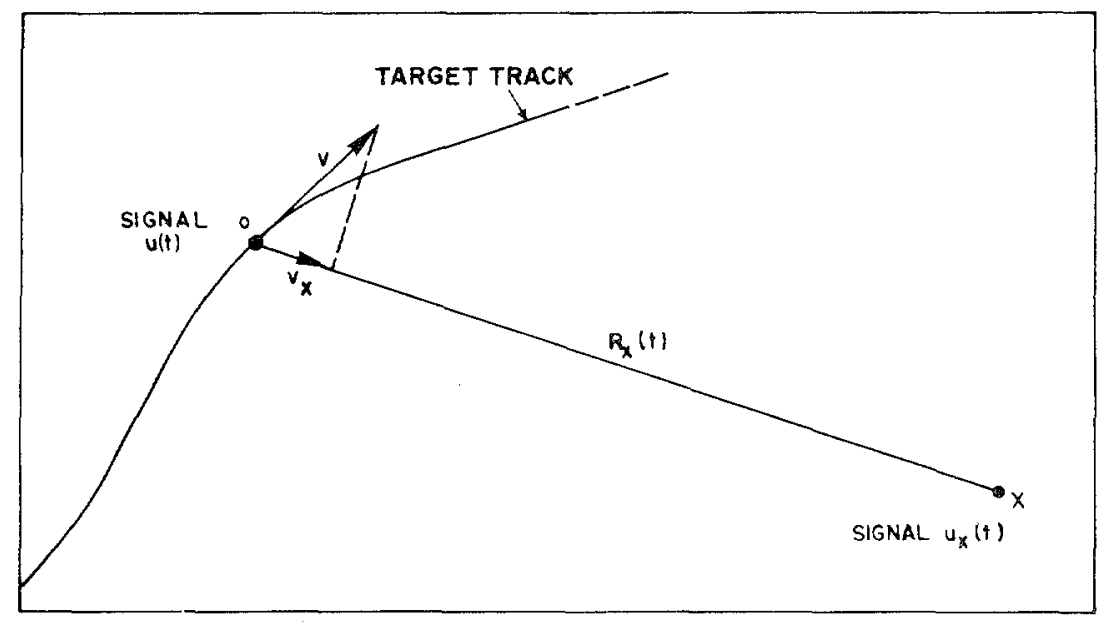

Fig. A-1. System geometry.

$$
d(k t)=k d t+t d k=\left(1+\frac{v_{x}}{c}\right) d t
$$

or

$$
k=1+\frac{1}{t} \int \frac{v_{x}}{c} d t .
$$

It is convenient to write $k(t)$ as $1+\delta(t)$ where $\delta(t)$ is the time scale-factor shift variable. Consequently,

$$
\delta=\frac{1}{t} \int \frac{v_{x}}{c} d t .
$$

The time scale-factor shift is, thus, a form of running time average of the function $v_{x} / c$.

\section{Time Delay Approach}

A second approach to the problem, which more clearly defines the boundary conditions, will now be presented.

Referring to Fig. A-1 and ignoring propagation loss, it is clear that the received signal $u_{x}(t)$ will be

$$
u_{x}(t)=u\left\{t-R_{x}(t) / c\right\}
$$

where $R_{x}(t)$ is the propagation range from the source to the receiving sensor and $c$ is the average propagation velocity along the transmission path.

From Newtonian mechanics, the range variable may be written as

$$
R_{x}(t)=R_{x}\left(t_{0}\right)-\int_{t_{0}}^{t} v_{x}(\tau) d \tau
$$

where $t_{0}$ represents an arbitrary time along the source motional track, and $R_{x}\left(t_{0}\right)$ is the state variable associated with the choice of the initial time $t_{0}$. The functional argument in the right-hand side of (A4) may therefore be written

$$
\begin{aligned}
k t-T_{0} & =(1+\delta) t-T_{0} \\
& =t+\int_{t_{0}}^{t} \frac{v_{x}}{c} d \tau-\frac{R_{x}\left(t_{0}\right)}{c} .
\end{aligned}
$$

Thus, it is evident that, if we ignore signal attenuation and delay time, a moving source signal $u(t)$ is transformed into a temporally distorted replica $u(k t)$ at the receiving sensor where

$$
k=1+\delta=1+\frac{1}{t} \int_{t_{0}}^{t} \frac{v_{x}}{c} d \tau .
$$

The choice of the initial time $t_{0}$ is arbitrary and is reflected in the time delay state variable $R_{x}\left(t_{0}\right) / c$. Stated another way, this implies that one may arbitrarily choose (or thumbtack) the initial reference time for the source function. This then, gives the time delay state $R_{x}\left(t_{0}\right) / c$ as an initial or boundary condition. Subsequently, no further change in this time delay value is required if one correctly utilizes the time scale factor variable. As a consequence, if the source dynamics or motional variables are known precisely beyond an initial time $t_{0}$, one may correlate any known source signal over an indefinite period of time without further need to re-reference the time delay alignment. The general form of the time scale-factor shift variable is simply

$$
\delta=\frac{1}{t} \int_{a}^{t} \frac{v_{x}}{c} d \tau
$$

where $a$ is an arbitrary constant.

\section{ACKNOWLEDGMENT}

The author gratefully acknowledges the assistance of W. L. Anderson in programming the data computations presented in this paper.

\section{REFERENCES}

[1] R. P. Flanagan et al., "Coherent analysis of ray propagation with moving source and fixed receiver," J. Acoust. Soc. Amer., vol. 56, pp. 1673-1686, Dec. 1974.

[2] E. C. Titchmarsh, Introduction to the Theory of Fourier Integrals. London, England: Oxford, 1937.

[3] G. A. Cambell and R. M. Foster, Fourier Integrals for Practical Applications. New York: Van Nostrand, 1948.

[4] E. C. Titchmarsh, The Zeta-Function of Riemann. Cambridge, England: Cambridge, 1930.

[5] G. N. Watson, A Treatise on the Theory of Bessel Functions, 2nd ed., Cambridge, England: Cambridge, 1958. 
[6] Y. L. Luke, "Some notes on integrals involving Bessel functions," J. Math. Phys., vol. 29, pp. 27-30, 1950.

[7] J. Boersma, "Computation of Fresnel integrals," Math. Comput., vol. 14, p. $380,1960$.

[8] T. Pearcey, Table of the Fresnel Integral. London, England: Cambridge, 1956.

[9] P. E. Green, Jr., "The output signal-to-noise ratio of correlation detectors," IRE Trans. Inform. Theory, vol. IT-3, pp. 10-18, Mar. 1957.
[10] W. B. Davenport, Jr. and W. L. Root, Introduction to Random Signals and Noise. New York: McGraw-Hill, 1958.

[11] A. A. Gerlach, Theory and Applications of Statistical WavePeriod Processing. New York: Gordon and Breach, 1970.

[12] M. Abramowitz and I. A. Stegun, Eds., Handbook of Mathematical Functions (Applied Mathematics Series 55). Washington, DC: NBS, June 1964.

[13] H. B. Dwight, Tables of Integrals and Other Mathematical Data. 4th ed., New York: Macmillan, 1961.

\title{
A Fast Fourier Transform (FFT) Based Sonar Signal Processor
}

\author{
ROBERT C. TRIDER
}

\begin{abstract}
The design for a convolution processor is presented, which employs a single highly parallel implementation of the fast Fourier transform (FFT) algorithm. This processor is eminently suited for real-time matched filtering of coded signals encountered in sonar systems. Computer simulations have shown that this processor, which uses fixed point arithmetic and modest word sizes, can efficiently handle signals with multiple targets and relatively large Doppler shifts. The parallel architecture provides a throughput rate sufficient for computing both forward and inverse transforms in the one processor. The system is flexible permitting frequency domain adaptive beamforming, attractive in many sonar applications.
\end{abstract}

\section{INTRODUCTION}

T HIS paper presents a design for a signal processing system which has the speed and versatility to handle a wide variety of underwater acoustic research systems. The design approach is novel in that a fixed point highly parallel fast Fourier transform (FFT) structure is employed. This approach differs from that reported in radar applications [1], [2] where a cascaded pipeline structure is used. The parallel design is feasible because of significant differences between sonar and radar signal processing requirements. The differences allow the same processor to be used in both forward and inverse transform modes and permit frequency domain techniques to be used in beamforming and signal filtering.

The basic requirements of the processor are beamforming (the combining of various element outputs in the hydrophone array to form directional receiving beams) and signal filtering. In passive sonar applications this filtering is primarily narrowband spectral analysis. Active systems also depend on similar spectral analysis when long $\mathrm{CW}$ tones are transmitted as in

Manuscript received February 14, 1977.

The author is with the Defence Research Establishment Atlantic Research \& Development Branch, Department of National Defence, Dartmouth, N.S., Canada.
Doppler sonars. In addition, when coded waveforms are used, such as linear frequency modulated (LFM) pulses, the processor must carry out matched filtering or correlation detection.

These same basic requirements are common to radar and sonar, but the practical differences are sufficient to support quite different processor design philosophies. For example, the time bandwidth (TW) products which can be supported by the underwater acoustic channel are approximately one order of magnitude less than employed in radar. A practical TW limit of about 200 is implied from various channel measurements [3]. Experiments have shown maximum coherence bandwidths of $200 \mathrm{~Hz}$ and coherence times of about $1 \mathrm{~s}$ to be typical of open ocean conditions at usual active sonar frequencies.

Another significant difference is the relatively large Doppler shifts encountered in sonar. Even with relatively Dopplertolerant signals such as LFM, several replicas must be used in the correlation or matched filter receiver to span the anticipated range of target speeds.

It has therefore been possible to exploit the advantages of a fast, parallel structured FFT as the central part of the processor. The inherent advantage of basing a general-purpose signal processor about an FFT is the simplification and processing freedom that exist when signals are in the frequency domain. Beamforming and matched filtering use a number of identical arithmetic operations. Likewise, beamforming and matched filtering coefficients are simple arrays of complex numbers which can be changed easily to handle different hydrophone arrays and transmitted waveforms.

Adaptive beamforming is also easily realized in the frequency domain through dynamic changes in the beamforming coefficients. Such adaptive techniques are of interest in sonar because of the frequency dependent directional characteristics shown by noise and reverberation.

In order to take advantage of the frequency domain in this 